\title{
AUTOMAÇÃO DOS PROCESSOS DE RECEBIMENTO, ARMAZENAMENTO E DISTRIBUIÇÃO DE COMBUSTÍVEIS*
}

\section{Resumo}

Cristiano Citi Ottoni ${ }^{1}$ Deivily Lage ${ }^{2}$

Diego Rafael Monteiro Diniz ${ }^{3}$

Fabrício Couto Carneiro 4

Vicente Magela dos Reis ${ }^{5}$

Os processos de recebimento, armazenamento e distribuição de combustíveis em grandes empresas mineradoras necessitam de um controle robusto e eficaz de todas as operações, uma vez que representam elevado custo por tonelada de minério produzida e expedida. Para tanto, a automatização dos processos que envolvem combustíveis é necessária, capaz de promover redução de custos, aumento de produtividade, confiabilidade e precisão nos dados e melhorias nas condições de trabalho na gestão e operação em combustíveis. Foi aplicada uma análise descritiva de um estudo de caso de um projeto de automação para esta finalidade implantado na empresa VALE S/A. Foi feita uma avaliação sistemática dos recursos, equipamentos e tecnologias necessárias a completa automação e integração dos processos que envolvem combustíveis e os ganhos alcançados após a implantação. Palavras-chave: Automação de processos; Combustíveis; Controle de processos industriais; Integração de sistemas de automação.

\section{AUTOMATION OF FUEL RECEIPT, STORAGE AND DISTRIBUTION PROCESSES Abstract}

The processes of receiving, storing and distributing fuels in large mining companies require a robust and efficient control of all operations, since they represent a high cost per ton of ore produced and shipped. To do so, automation of processes involving fuels is necessary, capable of promoting cost reduction, increased productivity, reliability and accuracy in data and improvements in working conditions in fuel management and operation. We applied a descriptive analysis of a case study of an automation project for this purpose implemented in the company VALE S / A. A systematic evaluation of the resources, equipment and technologies required for complete automation and integration of processes involving fuels and the gains achieved after the implementation.

Keywords: Fuels; Industrial process control; Integration of automation systems; Process automation.

1 Engenheiro de produção formado pela Fundação Comunitária de Ensino Superior de Itabira com especialização em automação industrial pela Universidade Federal de Minas Gerais, engenheiro de automação na gerência de automação, energia, telecomunicações e combustíveis, VALE S/A, Itabira, Minas Gerais, Brasil.

2 Engenheiro de produção formado pela Função Presidente Antonio Carlos, engenheiro de operações em combustíveis na gerência de automação, energia, telecomunicações e combustíveis, VALE S/A, Itabira, Minas Gerais, Brasil.

3 Engenheiro de controle e automação formado pela Universidade Federal de Ouro Preto, engenheiro de automação na gerência de automação, energia, telecomunicações e combustíveis, VALE S/A, Mariana, Minas Gerais, Brasil.

4 Administrador formado pela Faculdade Batista de Vitória, analista de automação na gerência de automação, energia, telecomunicações e combustíveis, VALE S/A, Itabira, Minas Gerais, Brasil.

5 Engenheiro eletricista formado pelo Centro Universitário do Leste de Minas Gerais, engenheiro de automação na gerência de automação, energia, telecomunicações e combustíveis, VALE S/A, Itabira, Minas Gerais, Brasil. 


\section{INTRODUÇÃO}

Com a grande demanda de consumo de diesel na VALE e com a necessidade de aumentar a integração e consolidação das informações entre nas etapas de recebimento, estocagem e distribuição com a necessidade de forte redução nos custos de gestão do combustível se faz a necessidade de automatizar o processo de recebimento, armazenamento e distribuição dos combustíveis, visando uma maior confiabilidade do processo de monitoramento, controle e auditoria. Esta automação é capaz de diminuir a possibilidade de falha humana durante o processo de operação de combustíveis devido à utilização de tecnologias aplicadas na aquisição, monitoramento e controle gerados nos postos de abastecimento de combustíveis.

No cenário anterior, o processo de descarga de carretas (recebimento) era realizado de forma manual, a validação do volume descarregado realizado através da marcação de uma seta (indicação visual). Caso a marcação observada estivesse abaixo da referência, a carreta recebia um complemento de combustível retirado do próprio estoque do posto de abastecimento a fim de se alcançar a marcação da seta em uma indicação conhecida na escala visual. Após este complemento, o volume de combustível existente da carreta era descarregado nos tanques e o volume descarregado era definido como a diferença entre o volume teórico da carreta de combustível e o complemento realizado. Esta etapa do recebimento é responsável pela validação do volume recebido e consequente validação do valor a ser desembolsado pela empresa no pagamento ao fornecedor.

O sistema de estoque e armazenamento de combustíveis também era controlado através de uma indicação visual por régua com seta de indicação do nível dos tanques de armazenamento. Através de uma tabela de arqueamento com pontos conhecidos (nível $x$ volume), era registrado o volume estocado em cada tanque conforme a indicação visual da régua.

O processo de abastecimento de combustíveis (distribuição) era controlado por um sistema existente gerenciado por uma empresa terceirizada responsável por realizar o controle e gestão dos abastecimentos realizados. No entanto, devido a baixa confiabilidade dos dados registrados pelo sistema, os abastecimentos também eram registrados manualmente pelos frentistas e operadores dos postos de abastecimento através dos valores indicados em registradoras mecânicas em blocos medidores por rodas ovais. O medidor de rodas ovais mantém as rodas engatadas e determina volumes de medição. O medidor possui necessariamente um comprimento com duas engrenagens que fazem girar as rodas, mantendo sempre uma parte dos seus perfis com um espaço mínimo. A pressão diferencial entre a montante e a jusante aplicadas as rodas ovais as faz girar [1].

Os dados gerados pelo sistema da empresa terceirizada eram integrados em um banco de dados próprio e constantemente eram confrontados pelos registros manuais para realização de correções na base de dados da empresa terceirizada. O banco de dados era de acesso restrito pela empresa terceirizada e a VALE recebia somente os relatórios com os controles de estoque, movimentação, abastecimento e recebimentos de combustíveis em toda a diretoria em sistema integrado de gestão empresarial ERP VALE, conforme ilustrado na figura 1. Este processo de gestão da distribuição terceirizado era responsável, em 2013, ano da concepção do projeto, por um custo em torno de $\mathrm{R} \$ 13 \mathrm{M} / \mathrm{ano}$ o que representava cerca de $40 \%$ do total do custo de toda a estrutura de gestão de combustíveis na diretoria. 


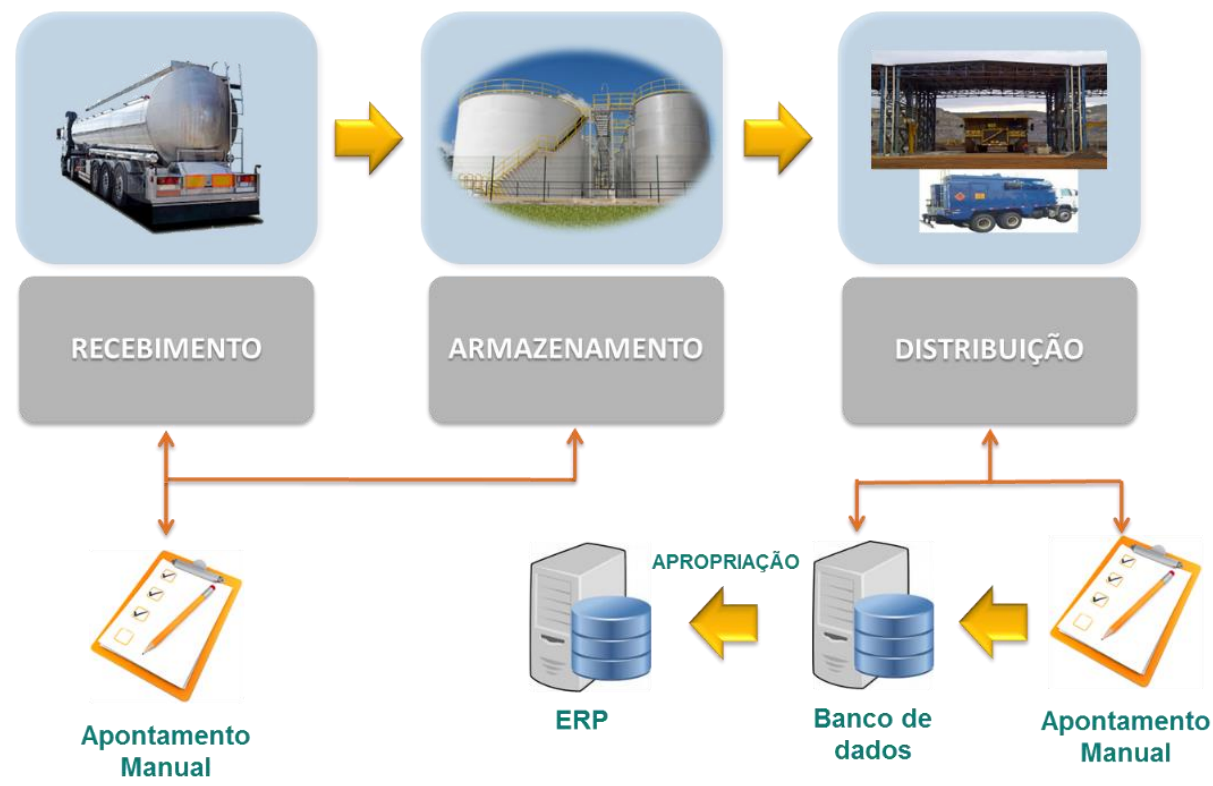

Figura 1. Fluxo de processo de gestão manual de combustíveis

Toda a planta industrial necessita de algum tipo de controlador para garantir uma operação segura e economicamente viável [2]. Um controle robusto e eficaz aplicado ao recebimentos, armazenamentos e distribuições de combustíveis é necessário visto que a quantidade de abastecimentos realizados diariamente em toda a diretoria é considerável e representam um elevado custo na composição de custos por tonelada de minério produzida e expedida.

Embora existam tamanhos e complexidades diferentes, todos os sistemas de controle podem ser divididos em três partes com funções bem definidas: Os transdutores (sensores), os controladores e os atuadores [2]. Os sensores são responsáveis por interagir de maneira direta ou indireta coma variável que se deseja medir, converter uma condição física de processo específica em um sinal elétrico modulado proporcional ao valor da variável que deseja medir, podendo este ser tratado por um controlador. Este, por sua vez, pode ser programado de tal forma que os dados e informações medidas sejam manipuladas, tratadas e aplicadas de diferentes meios. O controlador pode determinar as suas saídas que são conectadas a atuadores que convertem estas saídas em sinais elétricos equivalentes, podendo executar funções tais como: ligar/desligar um equipamento e abrir/fechar uma válvula.

Um tipo de controlador que é utilizado em grande escala em ambientes industriais são os CLPs (controladores lógicos programáveis). Um CLP é definido como um sistema eletrônico operando digitalmente, projetado para uso em um ambiente industrial, que usa uma memória programável para armazenagem interna de instruções orientadas para o usuário implementar funções específicas, tais como lógica sequencial, temporização, contagem e aritmética, para controlar, através de entradas e saídas digitais ou analógicas, vários tipos de máquinas ou processos [2].

A medição de variáveis de processo pode ser realizada através de instrumentos eletrônicos de medição e controle apropriados. Os instrumentos de medição e controle permitem manter e controlar estas variáveis em condições mais adequadas/precisas do que se elas fossem controladas manualmente por um operador [1]. 


\section{MATERIAIS E MÉTODOS}

Este trabalho é caracterizado como uma pesquisa descritiva, por se tratar de uma análise e correlação de fatos baseada em um estudo de caso aplicado. Por meio de pesquisas descritivas, procura-se descobrir com que frequência um fenômeno ocorre, sua natureza, suas características, causas, relações e conexões com outros fenômenos [3].

O estudo de caso em questão se trata da implantação de um sistema automático, associado a um sistema de controle e gestão totalmente integrado, customizado as necessidades operacionais para as etapas de recebimento, armazenamento e distribuição de combustíveis nas operações de ferrosos Sudeste da VALE S/A que contempla os complexos mineradores de Itabira, Mariana e Minas Centrais pertencentes ao estado de Minas Gerais.

O projeto implantando teve como objetivo a integração de informações, aumento da robustez na gestão e controle de movimentação e estoque de combustíveis, redução de perdas no processo, redução de exposição a atividades perigosas e forte redução no OPEX do processo de gestão, monitoramento e controle de combustíveis. A análise acerca do estudo de caso proposto será feita de forma descritiva, detalhando as características e ganhos proporcionados em cada processo de combustíveis, a integração dos processos e sua interface com as camadas de operação e gestão da empresa.

\section{RESULTADOS E DISCUSSÃO}

\subsection{Análise de viabilidade}

Para o estudo de viabilidade de implantação do projeto, foram analisados os dados de abastecimento de combustíveis, abastecimento de óleos lubrificantes, quantidade de postos de abastecimento fixos e móveis existentes por complexo minerador e o custo anual referente aos abastecimentos supracitados. Os dados foram levantados em 2013 identificando as prioridades, fragilidades e oportunidades de redução de custos nos processos envolvendo combustíveis e lubrificantes. A tabela 1 estabelece uma relação entre a quantidade de postos de abastecimento (fixos e móveis), frota de equipamentos e quantidade de abastecimentos realizados por ano.

Tabela 1. Dados levantados para definição de quantidade de abastecimentos por ano nas operações de ferrosos sudeste. Relação de postos fixos de abastecimento, posto móveis de abastecimento (comboios) e quantidades de equipamentos em operação.

\begin{tabular}{lcccc}
\hline & $\begin{array}{c}\text { Complexo } \\
\text { Itabira }\end{array}$ & $\begin{array}{c}\text { Complexo } \\
\text { Mariana }\end{array}$ & $\begin{array}{c}\text { Complexo } \\
\text { Minas } \\
\text { Centrais }\end{array}$ & $\begin{array}{c}\text { Operações } \\
\text { Ferrosos } \\
\text { Sudeste }\end{array}$ \\
\hline Posto Fixo & 3 & 4 & 3 & $\mathbf{1 0}$ \\
\hline Posto Móvel & 8 & 11 & 4 & $\mathbf{2 3}$ \\
\hline Lubrificante / Graxa & 3 & 6 & 2 & $\mathbf{1 1}$ \\
\hline Equipamentos ativos & 722 & 641 & 578 & $\mathbf{1 9 4 1}$ \\
\hline Abastecimentos / Ano & 64.000 & 77.000 & 60.500 & $\mathbf{2 0 1 . 5 0 0}$ \\
\hline
\end{tabular}

A figura 2 mostra o volume distribuído de combustíveis e óleos lubrificantes por ano nas operações de ferrosos sudeste. As operações de ferrosos sudeste da VALE possui um consumo de 147.000.000,00 de litros de óleo diesel S10 e S500 que geram um custo operacional de cerca de $R \$ 351.000 .000,00$. 
Volume Distribuído de Diesel (MM / ano)
Outros Produtos Distribuídos (Qtd/ ano)

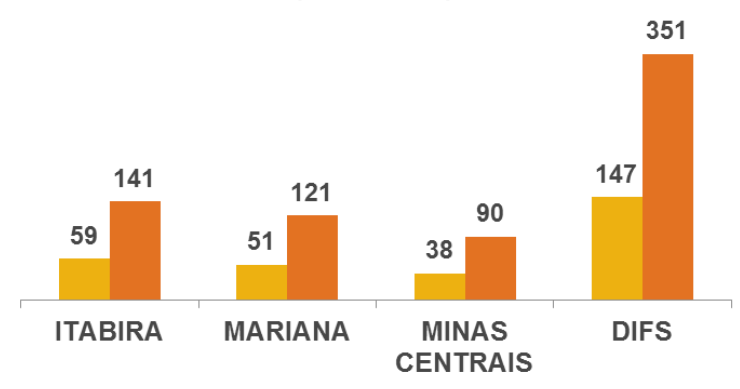

- Volume (Litros) $\quad$ valor (R\$)

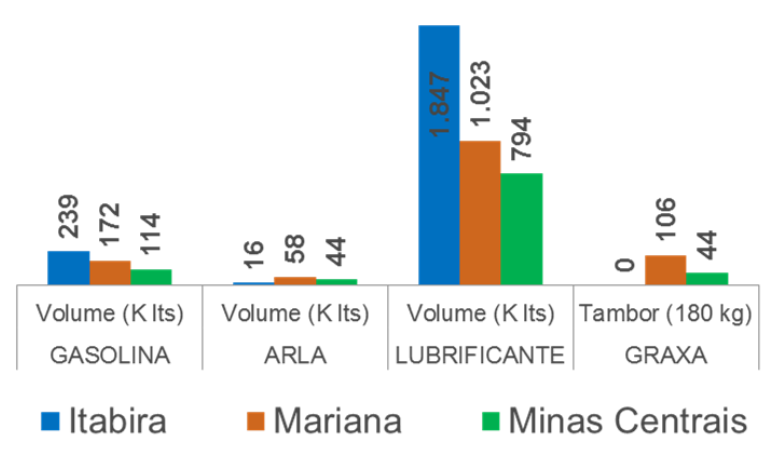

(b)

(a)

Figura 2. (a) Volume distribuído de diesel (MM/ano) / MM = Milhões. (b) Volume distribuído de outros produtos ( $\mathrm{K}$ litros) $/ \mathrm{K}=$ Milhares.

Com base nos dados apresentados e, através de uma análise de custo-benefício considerando os custos e equipamentos necessários para se realizar a automatização dos processos envolvendo combustíveis e lubrificantes, as operações de ferrosos sudeste optaram por priorizar a implantação do sistema automatizado para óleo diesel. No entanto, considerando que a infraestrutura de abastecimento de gasolina está integrada a infraestrutura de abastecimento de óleo diesel, foi incluído o escopo de gestão automática também para os processos de recebimento, armazenamento e distribuição de gasolina.

Conforme análise realizada em termos dos processos de recebimento, abastecimento e distribuição de combustíveis identificou-se que a maior fragilidade da gestão e controle manual de combustíveis existia no processo de recebimento dos mesmos, uma vez que o volume contido nas carretas era verificado através de uma marcação com seta visual com baixa precisão da medição dos dados. O processo de recebimento de combustíveis está intimamente ligado aos custos apresentados uma vez que nesta etapa, a VALE realiza o recebimento e o pagamento do volume contido em cada carreta para a distribuidora de combustíveis.

O processo de abastecimento, em contrapartida, não está intimamente ligado a magnitude dos custos apresentados na Figura 2, porém este deve ser robusto e eficaz de modo que os custos dos abastecimentos sejam distribuídos de forma correta e justa para todas as áreas clientes internas. O armazenamento de combustíveis, para o fluxo de processos de gestão e controle de combustíveis, poderá ser adotado como uma referência para análise de divergências tanto no recebimento quanto na distribuição de combustíveis, por se tratar de um processo intermediário em todo o fluxo.

A fim de se alcançar o controle necessário do recebimento, armazenamento e distribuição de combustíveis, vê-se necessária a adoção de um sistema de automação completo capaz de gerenciar os dados dos processos de combustíveis em tempo real com integração automática dos dados em banco de dados próprio para apropriação em sistema ERP. A figura 3 ilustra o fluxo do processo de recebimento, armazenamento e distribuição de combustíveis com a adoção de um sistema automático de gerenciamento e controle de todo o processo. 


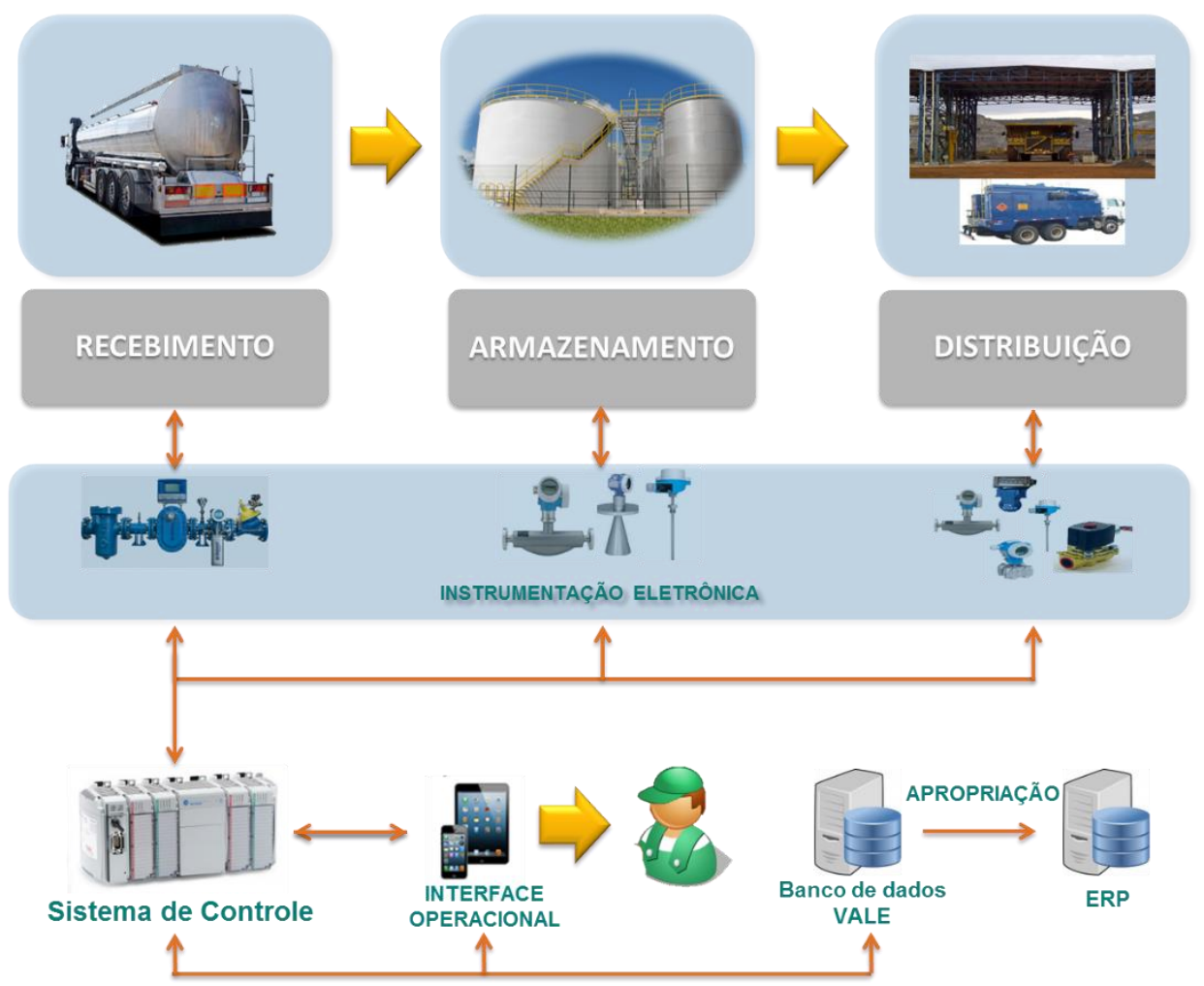

Figura 3. Fluxo de processo de gestão automática de combustíveis

Para se alcançar o objetivo estratégico do empreendimento, fez-se necessário a execução de um projeto multidisciplinar nas áreas de automação de processos, sistemas de informação, telecomunicações e gestão operacional de combustíveis capaz de proporcionar um valor presente líquido (VPL) positivo calculado em torno de $13 \%$ ao longo de 5 (cinco) anos de execução, incorporação de mão-de-obra direta e com infraestrutura necessária para sustentabilidade de toda a implantação realizada.

\subsection{Infraestrutura de rede e de automação}

Para a integração de todos os processos de combustíveis existentes, faz-se necessária a definição de uma infraestrutura de comunicação de todos os dispositivos de forma sólida, capaz de realizar e integração de todas as medições e estados de equipamentos de campo e as informações de todas as operações unitárias realizadas pelos frentistas e operadores dos postos de abastecimento.

Conforme ilustrado na figura 3 , as etapas de recebimento, armazenamento e distribuição deverão conter os instrumentos de medição adequados para medição das grandezas físicas vinculadas aos produtos em cada processo. No entanto, todas estas informações precisam ser processadas e tratadas em uma central única de processamento local e realizar a integração destes dados. Para esta tarefa, o CLP é altamente aplicável e o mesmo recebe e trata os sinais dos instrumentos de medição instalados e integra os sinais de entrada e saída discretos de campo (botoeiras de comando de bombas e válvulas, botoeiras de emergência, chaves de nível para indicação de nível muito alto em tanques de armazenamento, etc). O tratamento e as proteções de campo são realizados mediante uma programação de lógica específica para esta finalidade. É necessário que cada posto de abastecimento (seja ele fixo ou móvel) possua um CLP instalado com os módulos de entrada e saída necessários para integração dos sinais em campo e integrados em rede de automação. 
Para a utilização de interfaces de operação por dispositivos móveis em postos fixos de abastecimento, necessário que se tenha uma cobertura de rede wireless (sem fio) para comunicação dos dispositivos na rede, possibilitando que o operador utilize o sistema em campo. No caso de postos móveis, a integração em rede não é possível visto que as operações unitárias são realizadas na mina durante os turnos operacionais, onde não há cobertura da rede de automação específica para esta aplicação. Para este caso, cada posto móvel terá uma rede local responsável pela comunicação direta entre o CLP e o dispositivo móvel. Antes do encerramento do turno, o operador do posto móvel deverá se deslocar até um local com cobertura de rede wireless (postos fixos de abastecimento ou oficinas mecânicas) para sincronismo dos dados armazenados no dispositivo com a rede de automação.

\subsection{Automação do recebimento}

Conforme estudos realizados no mercado quanto a tecnologias e equipamentos para realização de recebimentos de combustíveis, adotou-se a utilização de SKIDs de descarga automática de combustíveis em postos de abastecimento que apresentassem, conforme sua capacidade e consumo diário, um ótimo custo/benefício para sua instalação. O SKID de descarga automática de combustíveis é um conjunto de equipamentos e instrumentos capaz de realizar o bombeamento do diesel contido no tanque da carreta de descarga para os tanques de armazenamento com um processo adequado de retirada de ar da linha de bombeamento, filtragem de impurezas que possam estar contidas no combustível, medição de temperatura, vazão e densidade e um computador industrial integrado capaz de realizar a totalização do volume descarregado com a sua conversão de valor a $20^{\circ} \mathrm{C}$.

Os SKIDs possuem certificação de calibração pela Rede de Calibração Brasileira (RBC) que é reconhecida pela distribuidora de combustíveis que fornece combustíveis para a VALE. Sendo assim, a totalização gerada em um processo de descarga é reconhecida por ambas as empresas, tornando-se o processo de recebimento mais confiável com medição de valores próximos da realidade do processo. No entanto, devido ao elevado custo de aquisição e instalação destes equipamentos, o mesmo foi aplicado somente para postos de abastecimento de equipamentos de mina $\mathrm{e}$ convencionais que possuem elevado consumo de óleo diesel. Para os demais postos de abastecimento, o processo se manteve da mesma forma, avaliando-se a divergência através da diferença de volume nos tanques de armazenamento.

Tabela 2. Estratificação de postos de abastecimento conforme a frota atendida.

\begin{tabular}{lcccc}
\hline & $\begin{array}{c}\text { Complexo } \\
\text { Itabira }\end{array}$ & $\begin{array}{c}\text { Complexo } \\
\text { Mariana }\end{array}$ & $\begin{array}{c}\text { Complexo } \\
\text { Minas } \\
\text { Centrais }\end{array}$ & $\begin{array}{c}\text { Operações } \\
\text { Ferrosos } \\
\text { Sudeste }\end{array}$ \\
\hline $\begin{array}{l}\text { Posto de abastecimento } \\
\text { (Equipamentos de Mina } \\
\text { convencionais) }\end{array}$ & 1 & 2 & 2 & 5 \\
\hline $\begin{array}{l}\text { Posto de abastecimento } \\
\text { (Equipamentos convencionais } \\
\text { somente) }\end{array}$ & 0 & 1 & 0 & 1 \\
\hline $\begin{array}{l}\text { Posto de abastecimento } \\
\text { (Equipamentos leves) }\end{array}$ & 2 & 1 & 1 & 4 \\
\hline Total & 3 & 4 & 3 & 10 \\
\hline
\end{tabular}




\subsection{Automação do armazenamento}

Para a etapa de armazenamento, foram adotados instrumentos de medição de nível do tipo radar para se medir o nível dos tanques de armazenamento de combustíveis em postos de abastecimento fixos com capacidade de conversão para volume em I (litros), conforme as características físicas de cada tanque e tipo (vertical ou horizontal).

Para postos de abastecimento móveis (comboios), a medição do volume no tanque é necessária somente no momento de troca de turno em que se realiza o processo de fechamento Físico x Físico e Físico x Contábil. Para este caso, foram adotados medidores de nível por pressão com capacidade de conversão para volume em I (litros), conforme as características físicas de cada tanque.

\subsection{Automação da distribuição}

Para a etapa de abastecimento, foram adotados instrumentos capazes de gerar pulsos elétricos conforme o movimento das rodas ovais contidas no bloco medidor mecânico de abastecimento tanto nos postos fixos quanto nos postos móveis de abastecimento. Os pulsos são regulados e configurados para garantir uma resolução definida de pulsos por litro para posterior conversão em totalização de volume abastecido no CLP.

Também foram integrados os sinais dos equipamentos de campo e demais sinais discretos necessários para a realização da operação de abastecimento. O CLP irá identificar, conforme a lógica de programação realizada, se um abastecimento está sendo realizado conforme a indicação de funcionamento das bombas de abastecimento.

\subsection{Interfaces operacionais}

Além das medições realizadas pelos instrumentos e dos sinais de campo advindos de equipamentos de campo, existem as operações de recebimento, movimentação, aferição e abastecimento que precisam ser realizadas no dia-a-dia da operação de cada posto de abastecimento.

Para tanto, foi desenvolvida uma aplicação específica para dispositivos móveis apropriados para esta aplicação pelos frentistas e operadores dos postos de abastecimento com a finalidade de se eliminar o preenchimento de formulários e check-lists operacionais adotados para as operações unitárias que são comumente utilizadas neste processo.

A aplicação específica é capaz de proporcionar uma interface amigável e prática com preenchimento automático de informações de processo (totalização de blocos medidores, volume de estoque atual, registro de volume abastecimento, recebido ou movimentado, etc) lidas diretamente do CLP do posto de abastecimento, possibilidade de leitura do código de barras do veículo para preenchimento automático dos dados do equipamento envolvido na operação de abastecimento ou movimentação, bastando apenas ao operador preencher manualmente algumas informações de campo relevantes para a operação unitária em andamento (medições de amostras de diesel, abertura ou fechamento de turno e horímetro dos equipamentos, por exemplo). Comandos e permissões para a realização de uma determinada operação unitária podem ser realizados por esta interface, tornando ainda mais prática e segura a execução das atividades pelos frentistas e operadores dos postos de abastecimento. 


\subsection{Integração automática de dados}

A figura 4 identifica de forma detalha o fluxo de integração dos dados em campo com os principais sistemas coorporativos.

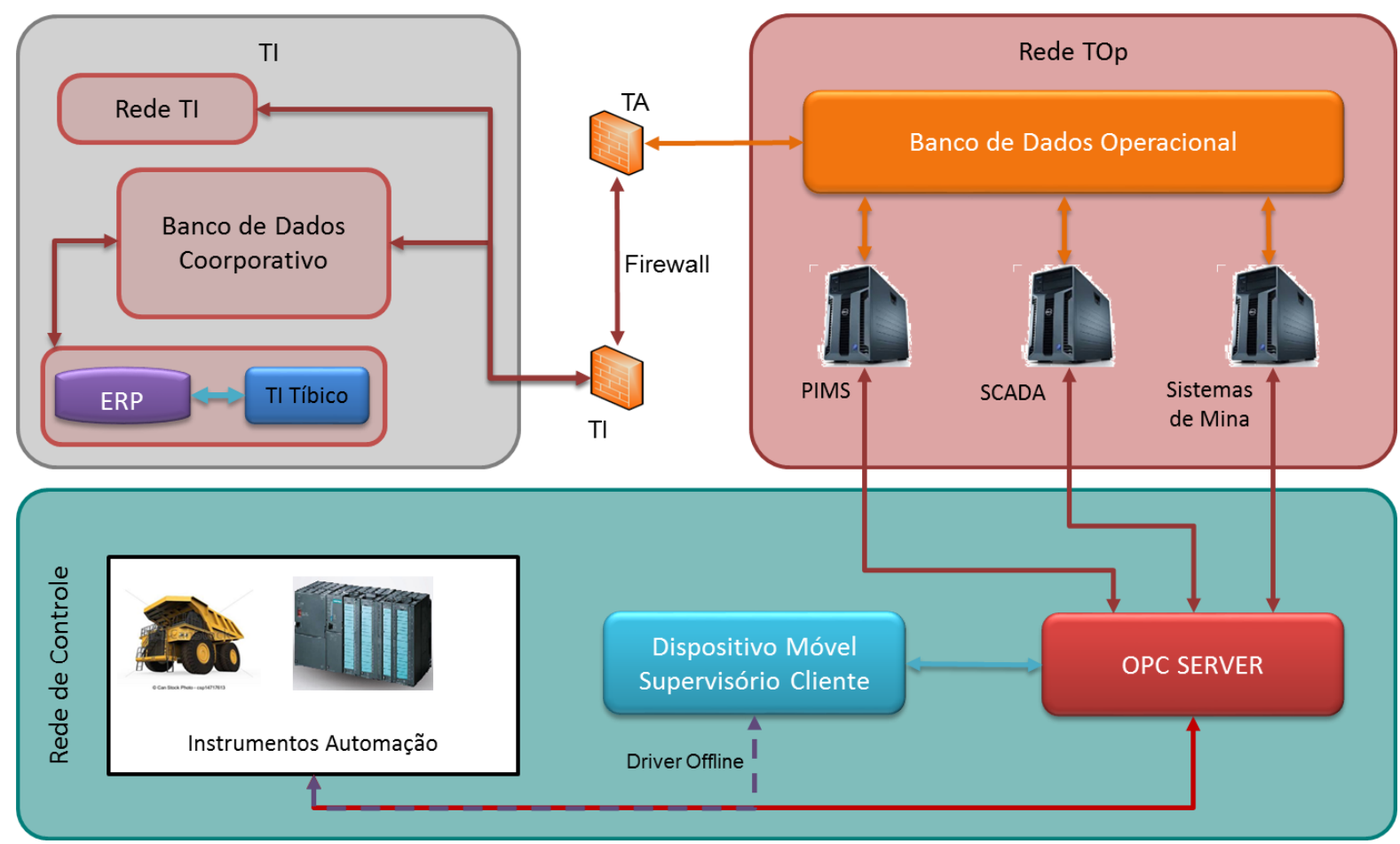

Figura 4. Fluxograma estruturado de integração do sistema automático de gestão e monotoramento em combustíveis.

Todos os dados e informações geradas em cada CLP em operação e pelas operações unitárias realizadas pelas interfaces de operação são integrados em um servidor OPC (Object linking and embedding for process control) responsável por realizar a leitura de todas as informações de campo e comunicar com as demais aplicações operacionais em rede TOp (tecnologia operacional VALE): PIMS (Sistema de monitoramento de informações de planta), SCADA (Sistema de supervisão e aquisição de dados) e sistemas de monitoramento e gestão de processos de mina. Todos estes sistemas são integrados no banco de dados operacional. Este banco é responsável por manter todos os dados e informações das operações unitárias realizadas em todos os postos de abastecimento da diretoria. Estes dados são utilizados para a realização das próximas operações unitárias pelas interfaces de operação e como base para alimentação de um banco de dados a nível coorporativo administrado pela área de tecnologia da informação (TI).

O banco de dados coorporativo é responsável por armazenar os mesmos dados gerados pelo banco de dados operacional, no entanto estes são tratados com a finalidade de se garantir a confiabilidade e rastreabilidade de todas as informações e dados acerca das operações unitárias realizadas no processo. A partir destes dados, os mesmos são integrados ao sistema ERP VALE e são gerados relatórios operacionais e gerenciais com informações confiáveis e íntegras a respeito de todo o processo, que podem ser utilizados pelas áreas operacionais e estratégicas da empresa. 


\section{CONCLUSÃO}

O projeto foi desenvolvido aliando a grande experiência das áreas de gestão de combustíveis e de automação das operações de ferrosos sudeste que se uniram no desafio de reduzir o custo da gestão do insumo, aumentar a robustez nos controles e trilhas de auditoria e reduzir os riscos associados às operações de recebimento, armazenamento e distribuição de combustíveis. Pensado e desenvolvido para atender ao processo VALE na diretoria e com as particularidades da empresa na gestão de combustíveis ao máximo da sua plenitude acabou por apresentar um sólido e comprovado resultado, proporcionando:

- Identificação, validação e liberação automática em todas as etapas do recebimento e distribuição de combustível (reduz o risco de manuseio indevido do diesel);

- Eliminação da necessidade de preenchimentos manuais de formulários e check-lists adotados para as principais operações unitárias realizadas nos processos que envolvam combustíveis;

- Execução do fechamento e divergência físico x contábil ao final de cada turno de maneira automática;

- Execução do fechamento físico x físico automática;

- Automação, controle e gestão mais robusta em todas as etapas e atividades do processo;

- Recuperação de perdas e sobras no recebimento.

O projeto implantado possui grande capacidade de expansão e aplicabilidade em outras áreas, sendo elas:

- Projeto desenvolvido preparado para receber outros insumos como lubrificantes, reagentes e outros. Podendo ser aplicado em qualquer unidade da VALE que utilize destes;

- $\quad$ Projeto desenvolvido para aplicação em qualquer unidade VALE nos processos de insumo que desejar.

\section{REFERÊNCIAS}

1 BEGA, E. A; BULGARELLI, R.; COHN, P. E.; DELMEÉ, G. J.; FINKEL, V. S.; KOCH, R. Instrumentação Industrial. 3aㅡ Ed. São Paulo: Editora Interciência. 2011, 694 p.

2 FRANCHI, C. M.; CAMARGO, V. L. A. de. Controladores Lógicos Programáveis Sistemas Discretos. 2ª Ed. São Paulo: Editora Érica Ltda. 2013, 352 p.

3 BARROS, A. J. da S.; LRHFELD, N. A. de S. Fundamentos de Metodologia Científica: um guia para iniciação científica. 2ª Ed. São Paulo: MAKRON Books, 2000, 182 p. 